\title{
Evaluation of the Efficacy and Safety of Percutaneous Biopsy of Lung
}

\author{
Sean E. McSweeney, Kevin N. O’Regan, Patrick D. Mc Laughlin, Lee Crush \\ and Michael M. Maher*
}

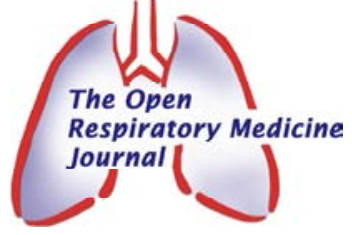

\author{
Department of Radiology, Cork University Hospital and University College Cork, Cork, Ireland
}

\begin{abstract}
Introduction: Percutanous needle biopsy of the lung (PNBL), under image guidance, has established itself as a safe and effective minimally-invasive method of obtaining a tissue diagnosis of pulmonary lesions, for selected patients with suspected pathologic processes. The purpose of this study was to evaluate the diagnostic yield and safety of percutaneous core biopsy of the lung (PCBL) without Fine Needle Aspiration Biopsy (FNAB), with specific attention to potential risk factors that may predict post-biopsy pneumothorax.
\end{abstract}

Materials and Methods: A retrospective analysis of 75 consecutive PCBL procedures between January 2006 to March 2008 involving 72 patients with a documented pulmonary nodule or mass lesion on CT scan of Thorax. The study population included 38 males (52.8\%) and 34 females (47.2\%) aged 20-85 years (mean age 63.6 years). A co-axial cutting system with a 19-gauge outer needle and a 20-gauge inner automated cutting needle (Temno, Allegiance Healthcare Corporation, Ohio, USA) was used in all patients.

Results: Lesions varied in size from $0.7 \mathrm{~cm}$ to $10.7 \mathrm{~cm}$ (mean maximum trans-axial diameter $3.1 \mathrm{~cm}$ ). The mean number of core biopsy samples obtained was 3.1 (range 1-5). Of the 75 PCBL procedures, 71 yielded specimens adequate for histopathologic evaluation, consistent with a technical success rate of $95 \%$. Malignancy was detected in 48 specimens (64\%) and benign diagnoses were identified in 23 specimens (31\%). As mentioned, 4 specimens (5\%) were nondiagnostic. The most common biopsy-induced complication was pneumothorax, occurring in 15 patients (20\%), with 4 $(5.3 \%)$ requiring thoracostomy tube placement.

Conclusion: PCBL without FNAB, under CT fluoroscopy guidance, has an excellent diagnostic accuracy in obtaining a conclusive histologic diagnosis of thoracic lesions and is comparable to FNAB in terms of safety and rates of occurence of complications.

Keywords: Percutaneous biopsy of lung, non-fine needle, efficacy, safety.

\section{INTRODUCTION}

In the United States and Europe, lung cancer is the leading cause of cancer-related deaths, with a 5-year survival rate of $50 \%[1,2]$. With advances in computed tomography (CT) technology and its widespread application in clinical practice, dectection of lung lesions in the population has increased in recent years. Percutanous needle biopsy of the lung (PNBL), under image guidance, has established itself as a safe and effective minimally-invasive method of obtaining a tissue diagnosis of pulmonary lesions, for selected patients with suspected pathologic processes [3]. In parallel with refinements in imaging technology, biopsy equipment and technique, the yield of PNBL has also improved [4-7]. PNBL offers advantages over bronchoscopic biopsy particularly when lesions are peripheral and less than $2 \mathrm{~cm}$ in diameter.

When sampling pulmonary lesions, there is considerable inter-institutional variability in the technical performance of PNBL, some performing CT-guided fine needle aspriation

*Address correspondence to this author at the Department of Radiology, Cork University Hospital, Wilton, Cork, Ireland; Tel: +00 35386 1731929; Fax: +00 35321 4922857; E-mail: M.Maher@ucc.ie biopsy (FNAB) for cytologic evaluation, with others choosing percutaneous core biopsy of the lung (PCBL), and others a combination of both [8]. Typically, PCBL involves insertion of a hollow large bore needle, with a specially adapted cutting mechanism, into an organ to extract of piece of tissue for histologic evaluation [3]. While technically demanding, extensive experience documents PCBL as a safe and accurate procedure with limited morbidity and rare mortality [3, 8-10]. When compared with FNAB, PCBL seems to achieve a superior diagnostic accuracy for benign lung lesions (71-97\%) and a similarly high diagnostic accuracy for malignant lung tumours (88-95\%) [7, 11-12], with comparable complication risk and without the need for on on-site cytopathology [11-14].

The purpose of this study was to evaluate the diagnostic yield and safety of PCBL in our department, with specific attention to potential risk factors that may predict postbiopsy pneumothorax.

\section{MATERIALS AND METHODS}

Study population: From January 2006 to March 2008, 75 consecutive PCBL were performed on 72 patients with a documented pulmonary nodule or mass lesion on CT scan of Thorax in all cases. Six patients $(8.3 \%)$ had a PET CT 
subsequent to the original CT study. The study population included 38 males (52.8\%) and 34 females (47.2\%) aged 2085 years (mean age 63.6 years). Prior to each procedure, the risks and benefits were explained and written informed consent was obtained from each patient.

Fifteen patients $(21 \%)$ were asymptomatic at presentation, with a lesion identified incidentally on radiological imaging, but the majority of patients (57 patients, 79\%) were symptomatic. While 34 patients (47\%) described fatigue and shortness of breath, 12 (17\%) haemoptysis, and 26 (36\%) weight loss, only 5 (7\%) described all three symptoms. Sixty-one $(85 \%)$ patients had a smoking history, with 30 current smokers, 8 being exsmokers for less than a year, and 23 being ex-smokers for over a year. There was a known clinical history of chronic obstructive pulmonary disease (COPD) or emphysema in 23 patients (32\%), a known malignancy elsewhere in 14 (19\%), and a family history of lung cancer in $6(8 \%)$.

Prior to biopsy, each patient underwent a diagnostic noncontrast CT examination of the thorax with the institution's interventional CT scanner (Somatom, Siemens Medical Systems, Erlangen, Germany), in quiet respiration, to identify the lesion to be biopsied.

PCBL Technique: All biopsies were performed by an attending radiologist, who sub-specialises in thoracic radiology, (M.M.M.) with 15 years of experience. Every PCBL case was performed under conscious sedation using CT fluoroscopy as image guidance [15]. Contrast media was not used at fluoroscopy. Procedures were performed with the patient in a prone (44 patients), supine (27 patients), or lateral decubitus (4 patients) position, depending on the location of the lesion. Choice of patient position was based on achieving the most direct route for biopsy, that traversed the least amount of aerated lung and avoided bullae and fissures [16]. A standard procedure employing a co-axial cutting system with a 19 -gauge outer needle and a 20 -gauge inner automated cutting needle (Temno, Allegiance Healthcare Corporation, Ohio, USA) was used in all patients, allowing multiple samples to be taken following a single pleural puncture (see Figs. 1, 2A-D). The automated springdriven cutting needle allows a higher quality core specimen for histopathologic analysis to be obtained more easily [4]. The number of samples (core biopsies) acquired was recorded, as was the length of the needle used. Following pathological evaluation, the histologic reports were reviewed for final histological diagnosis. FNAB was not employed in any case.

After the procedure, patients were placed in the puncture side down position and observed. Chest radiographs were obtained at 1 and 3 hours post-PCBL to evaluate for the presence of pneumothorax. The frequency of pneumothorax, chest tube placement, and other complications was recorded. When required all thoracostomy tubes were inserted by a radiologist in the radiology department.

Statistical analysis was performed using commercial software (SPSS version 14.0). Fisher's exact test and Chisquared test were used to assess the statistical significance comparing patient risk factors with biopsy diagnosis and procedure complications. A probability value of $<0.05$ was considered to indicate statistical significance.

A Spearman correlation test was performed between current or former smoking status, known clinical history of COPD or emphysema, and magnitude of lesion depth from pleura with pneumothorax risk following PCBL.

Institutional Review board approval was obtained for this retrospective study and requirement for patient consent was waived.

\section{RESULTS}

Three patients underwent a second lung biopsy within the study period, and for the purpose of calculations, a repeat biopsy was considered a separate procedure.

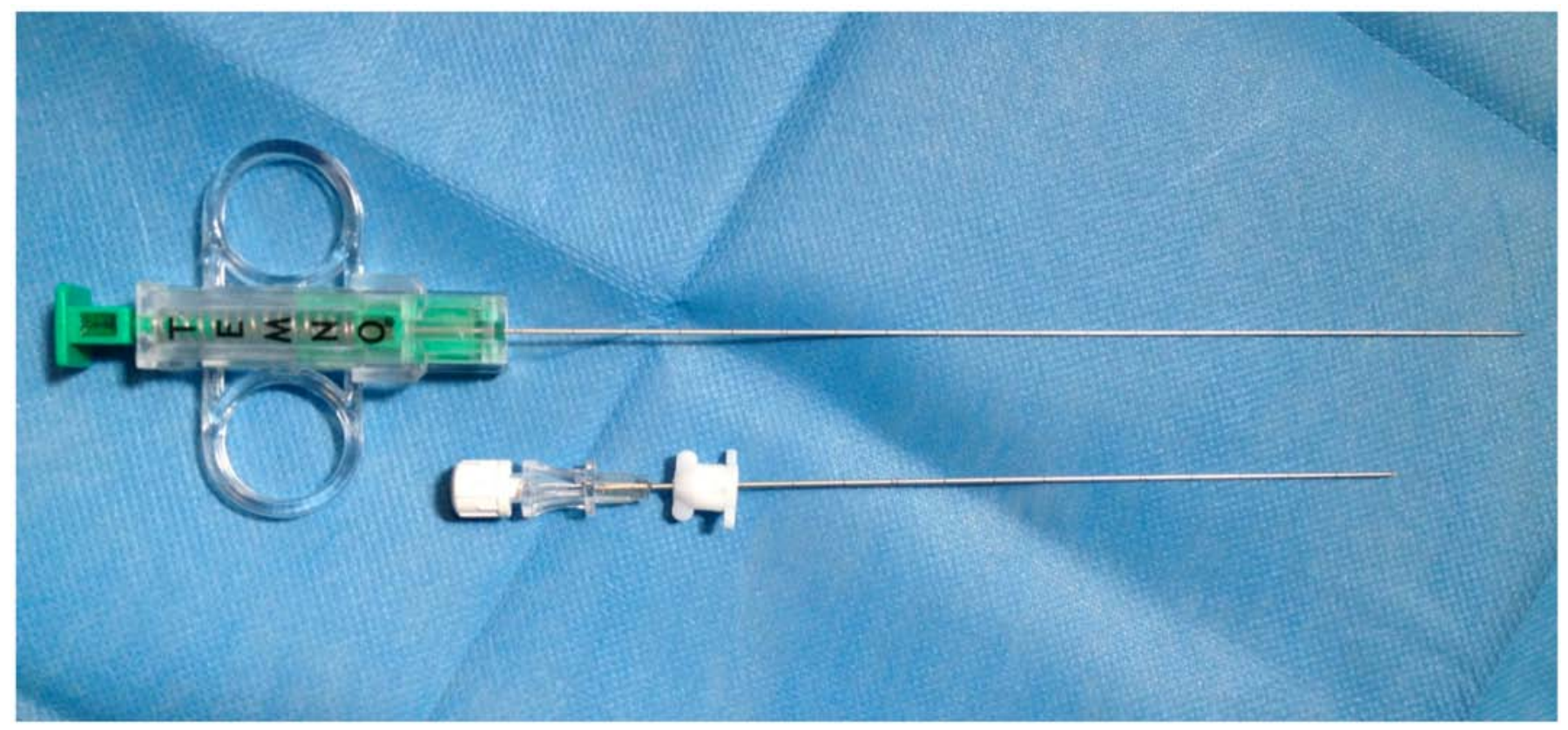

Fig. (1). 19 gauge/20 gauge TEMNO co-axial cutting system needle (Allegiance Healthcare Corporation, Ohio, USA). 


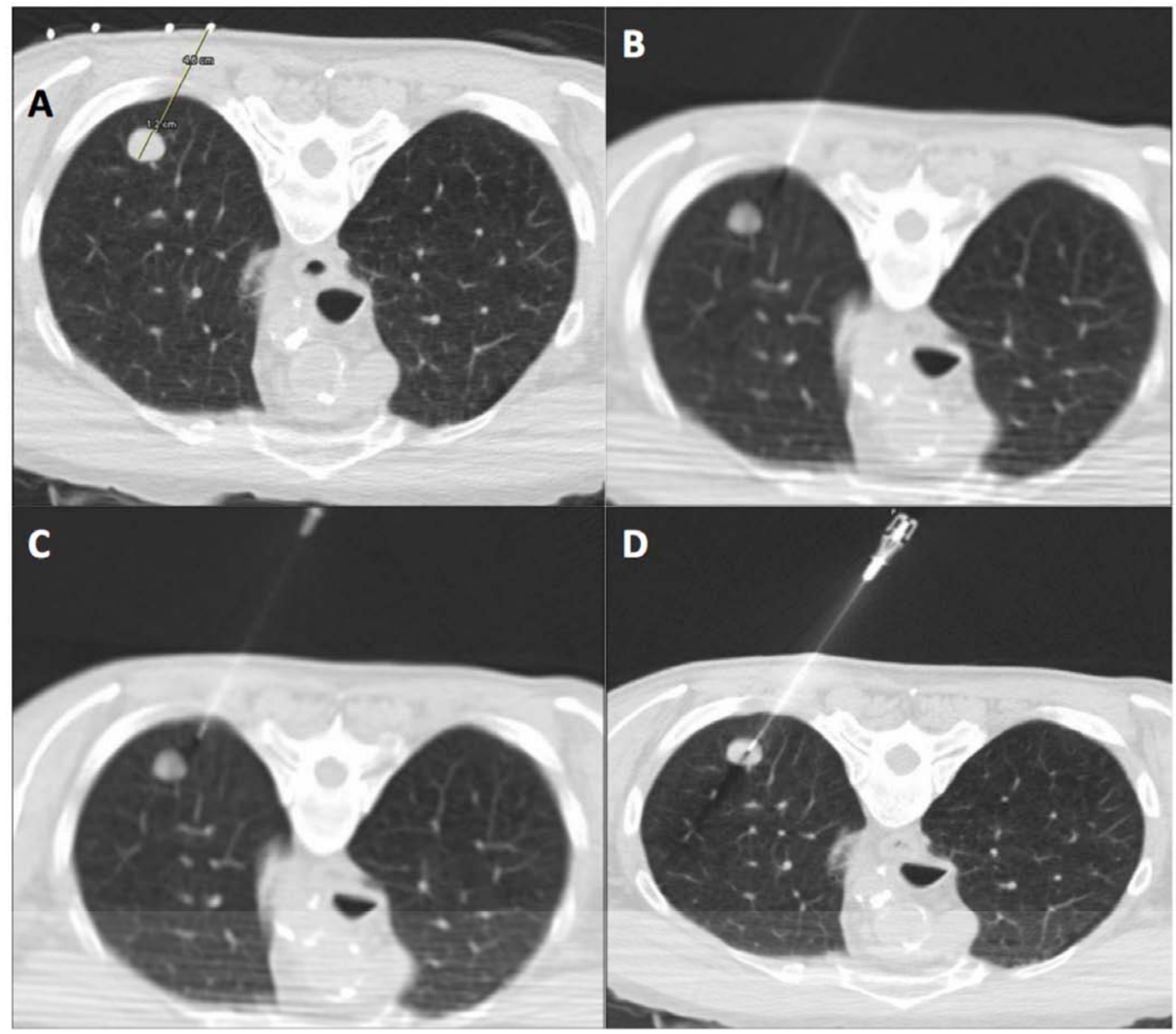

Fig. (2). CT guided percutaneous core biopsy of lung (PCBL). (A) Lesion is localized and access route is planned using thin section CT. The skin entry site is marked on the patient with indelible ink with use of CT gantry laser light. The distance to the lesion and depth of lesion is measured. (B) The introducer (19 gauge ultra-thin needle) is carefully aligned in the axial plane with guidance from CT gantry light. The needle is advanced in small increments through the soft tissue of the chest wall. (C) Once the introducer needle is correctly aligned, a single deliberate puncture of the pleura is made and the needle is advanced beyond the pleura for at least $2 \mathrm{~cm}$. If needle is misaligned, the introducer can be repositioned in small increments without exiting the lung. (D) Once satisfactory needle position is confirmed by CT, the introducer needle is advanced into the edge of the lesion. The inner stylet of the 19 gauge needle is removed and core biopsy performed with 20 gauge automated cutting needle.

The lesions varied in size from $0.7 \mathrm{~cm}$ to $10.7 \mathrm{~cm}$ (mean maximum trans-axial diameter $3.1 \mathrm{~cm}$ ). The mean depth of the lesion from the skin was $6.9 \mathrm{~cm}$ (range $2.8-12 \mathrm{~cm}$ ) and from the pleura was $2.5 \mathrm{~cm}$ (range $0-7.8 \mathrm{~cm}$ ). One of three lengths of co-axial cutting needle was used for biopsy, depending on lesion depth from pleural surface: $6 \mathrm{~cm}$ needle length in 5 procedures, $10 \mathrm{~cm}$ in 47 procedures, and $15 \mathrm{~cm}$ in 23 procedures. The mean number of core biopsy samples obtained was 3.1 (range 1-5).

Of the 75 PCBL procedures, 71 yielded specimens adequate for histopathologic evaluation, consistent with a technical success rate of $95 \%$. Malignancy was detected in 48 specimens (64\%) (see Table 1) while a benign diagnosis was identified in 23 specimens (31\%) (see Table 2). Four PCBL's were considered non-diagnostic following review by histopathology and discussion at the lung cancer multidisciplinary team meeting.

The most common biopsy-induced complications observed were pneumothoraces, occurring in 15 patients (20\%), with 4 (5.3\%) requiring thoracostomy tube placement. There was a low threshold for thoracostomy, with tubes placed immediately after biopsy in 2 patients with 
known severe emphysema despite only having small pneumothoraces (see Fig. 3A-C). There was increased perilesional airspace attenuation post-biopsy in 12 patients $(16 \%)$, indicating lung parenchymal haemorrhage (see Fig. 4A, B). Small volume haemoptysis, which was self limited in all cases, was reported by three patients $(4 \%)$, but there was no incidence of significant haemoptysis or haemothorax.

Table 1. Summary of Maligant Pathological Diagnosis on PCBL

\begin{tabular}{|c|c|}
\hline \multicolumn{2}{|c|}{ Biopsy Result: Malignancy $(\mathbf{n}=\mathbf{4 8})$} \\
\hline Pathological Diagnosis & Number (\%) \\
\hline \hline Non Small Cell Lung Cancer & $34(70.8 \%)$ \\
\hline Small Cell Lung Cancer & $4(8.3 \%)$ \\
\hline Lymphoma & $4(8.3 \%)$ \\
\hline Mesothelioma & $1(2.1 \%)$ \\
\hline Metastatic - Breast Primary & $3(6.2 \%)$ \\
\hline Metastatic - Colon Primary & $1(2.1 \%)$ \\
\hline Metastatic - Melanoma Primary & $1(2.1 \%)$ \\
\hline
\end{tabular}

Table 2. Breakdown of Benign PCBL Findings

\begin{tabular}{|c|c|}
\hline \multicolumn{2}{|c|}{ Biopsy Result: Benign $(\mathbf{n = 2 3})$} \\
\hline Pathological Diagnosis & Number (\%) \\
\hline \hline Fibrosis & $3(13.1 \%)$ \\
\hline Inflammation & $2(8.7 \%)$ \\
\hline Fungal infection & $2(8.7 \%)$ \\
\hline Hamartoma & $2(8.7 \%)$ \\
\hline Tuberculosis granulomatous inflammation & $2(8.7 \%)$ \\
\hline Vasculitis & $2(8.7 \%)$ \\
\hline Pneumoconiosis & $1(4.3 \%)$ \\
\hline Pleural Fibroma & $1(4.3 \%)$ \\
\hline Other & $8(34.8 \%)$ \\
\hline
\end{tabular}

Current or former smoking status, known clinical history of COPD or emphysema, and magnitude of lesion depth from pleura did not correlate statistically with increased pneumothorax risk following PCBL. There was a significant positive association between a malignant histologic diagnosis and a history of being a current or former smoker $(\mathrm{p}=0.037)$ [17].

\section{DISCUSSION}

CT-guided percutaneous transthoracic lung biopsy is a well-established, effective, and safe technique for the sampling of lung lesions to obtain a tissue diagnosis [3]. Bronchoscopy with direct examination of the visible airways is commonly the preferred invasive diagnostic procedure in the evaluation of lung malignancy. Diagnostic yield is higher and complication rates are lower with bronchoscopy when lesions are central or endobronchial. PNBL offers advantages when lesions are peripheral and also when they are less than $2 \mathrm{~cm}$ in diameter.

It is clinically important to make the distinction between malignant and benign disease, between primary and secondary neoplasms, and between specific cell types in the context of malignant disease, as this has a bearing on treatment selection and implications for prognosis. The relative success of a biopsy can be measured in terms of high diagnostic accuracy and a low rate of complications [3]. Many experts in the field suggest that PNBL will have higher technical success rates with low incidence of complications, when the procedure is well-planned and performed by a team which includes a radiologist, a technologist and nursing staff who are trained in all aspects of PNBL and who perform this procedure on a regular basis.

The preferences of individual radiologists with regard to biopsy technique appear to vary both between and within institutions [8]. When given the hypothetical case of a $3 \mathrm{~cm}$ lung mass located $1 \mathrm{~cm}$ from the pleural surface in the right lower lobe, the majority of questioned Members of the Society of Thoracic Radiology opted for FNAB as their tehnique of choice (73\%), while only $14 \%$ selected core biopsy [18]. Factors which influence preference for FNAB over PCBL are uncertain [8]. In this study, PNBL consisted of core biopsy (PCBL) only, without FNAB is all 75 procedures.

In this study, the overall diagnostic accuracy of specimens obtained by 75 consecutive PCBL procedures was 95\%. This compares favourably with reported success rates, which range from 77-96\% [5, 6, 19-24] and is well above the Society of Inteventional Radiology Guidelines suggested threshold yield of 75\% [3]. The use of CT fluoroscopy in all cases and the relative operator experience are both likely to have contributed to this high success rate [25]. In our experience, the use of CT fluoroscopy seems to have the advantage of shortening the length of the procedure and, vitally, the total time the biopsy needle remains within the patient's lung [4]. PCBL without FNAB (and on-site cytologist review) also reduces the length of the procedure and the time the biopsy needle spends in the lung, compared to FNAB as the time required for preparing and analysing the cytology slides is not reqired for PCBL alone. Shortening of these procedures has numerous potential advantages including reduced duration the patient remains sedated, leading to potentially reduced dosages of sedative and analgesic drugs (which is very important in these patients who often have numerous co-morbidities), and more efficient usage of a CT scanner.

As mentioned, 6 patients had a PET CT prior to biopsy. Of these, 4 patients had fluorodeoxyglucose (FDG) avid lung nodules or masses. The remaing two patients had pulmonary nodules, which were not FDG avid, measuring less that 1.0 $\mathrm{cm}$ (average $0.7 \mathrm{~cm}$ ), and were subsequently proven malignant on biopsy. The low rate of utilization of PET CT in our patient cohort, is explained by the fact that PET CT was not readily available to our patients during the study period. However, due to limitations of PET CT in definitive characterisation of lung nodules as malignant or benign, we follow a policy of performing PCBL in all nodules where PCBL is technically feasible and where the patient is considered fit to undergo PCBL. 


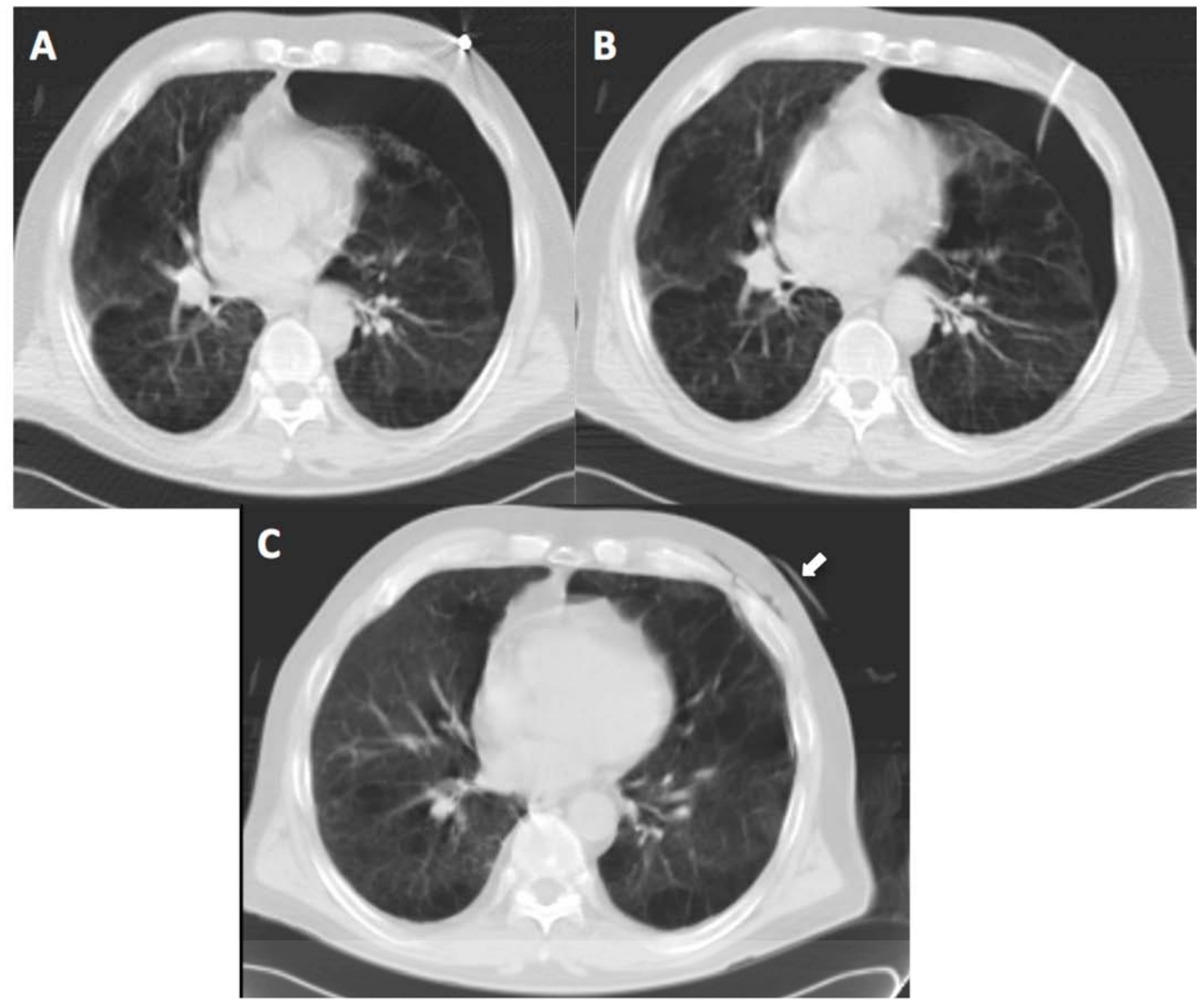

Fig. (3). Pneumothorax post biopsy. (A) CT post biopsy shows a small left pneumothorax in a patient with severe emphysema. (B) CT post insertion of small calibre chest tube with $(\mathbf{C})$ successful reinflation of left lung (the chest tube remains in situ and can be seen anteriolateral to the chest, white arrow).

The specimens obtained in 4 biopsies yielded inconclusive pathological results. These patients went on to have further radiological follow-up and repeat biopies (including Video-Assisted Thoracoscopic Surgery (VATS) biospy) as clinically indicated. The biopsy specimens of 8 lesions showed absence of malignant cells and the presence of inflammatory cells. Consensus agreement of the radiologist, the referring physician and the pathologist that the samples obtained were of adequate volume and quality and were sufficiently representative of the lesion in question meant that these specimens were conclusively deemed benign and did not warrant re-biopsy. These patients were followed clinically by the referring respiratory physician and by imaging in all cases and none proved malignant on longterm follow-up.

The most common complication observed was that of pneumothorax, consistent with previous studies of CTguided lung biopsy [3, 9, 16]. Reported pneumothorax frequency ranges from 12 to $45 \%$, seen more commonly in emphysematous patients, lesions proximal to lung fissures, and in cases requiring multiple pleural punctures $[13,16,21$, 26-28], with $2-15 \%$ requiring thoracostomy tube placement $[3,14,16,20-21,29-33]$. In this study, the incidence of pneumothorax was $20 \%$ which is comparable to quoted rates with requirement for thoracostomy tube placement in $5.3 \%$ of cases [3]. Though $5.3 \%$ of patients required thoracostomy tube placement, the low threshold for tube insertion in the context of severe known emphysema meant that half of these patients actually had small pneumothoraces, with tube insertion completed by the performing radiologist at the time of PCBL, mainly to prevent any deterioration. Overall, there was no incidence of any major complication or death.

The retrospective nature of this study, with a single common operator performing all procedures in a standardised manner, limits bias allowing the success and complications of PCBL to be assessed accurately. As a 


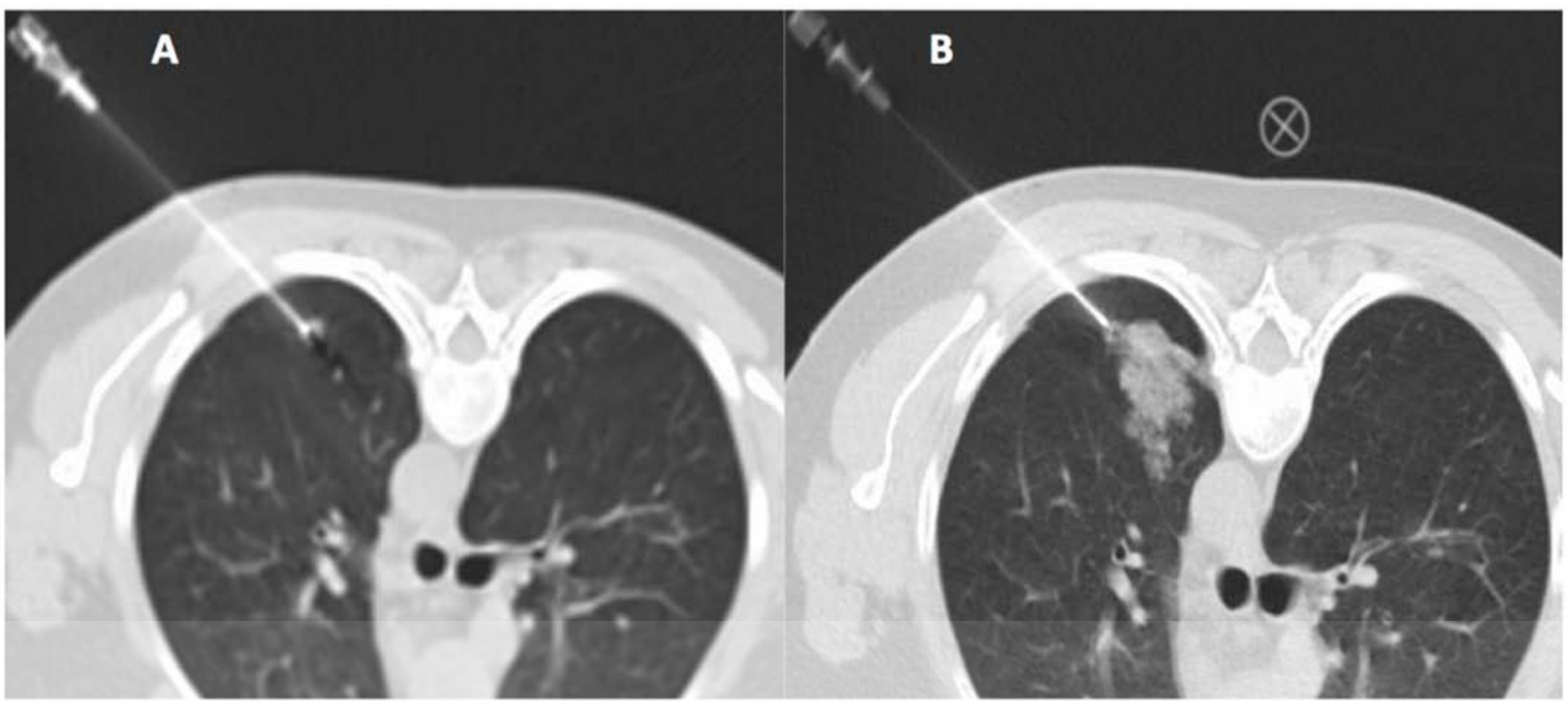

Fig. (4). Lung parenchymal haemorrhage. (A) Pre and (B) post biopsy CT in the same patient showing evidence of lung parenchymal haemorrhage (areas of air space attenuation around lesion). As in this case, the majority of patients did not experience haemoptysis.

consequence however, the study population, though representative, is relatively small. Another limitation of this study, potentially leading to bias, which is common to most other studies of PCBL, is that patients with known severe emphysema, very abnormal lung function, or a poor performance status, may have been selected out of this population, as PNBL could have been considered to be contraindicated due to unacceptable risk. Evaluation for pneumothorax was by means of chest radiographs at 1 and 3 hours post-procedure, as well as clinical observation for deterioration in respiratory function. It is possible that delayed clinically silent pneumothoraces, not evident on these two radiographs, may not have been detected prior to or after hospital discharge. However, in the study cohort, there were no readmissions to hospital for management of pneumothorax or other complications of PNBL. We did not encounter any cases of pleural dissemination of malignancy directly related to PNBL but larger studies would be required to fully determine the rates of this delayed complication of PNBL. Other limitations of our technique include that we did not strictly enforce a rapid needle-out patient roll over time (defined as a time of less than 10 seconds between removal of the biopsy needle and placing the patient biopsy-side down) which has recently been shown to result in a significantly less pneumothorax rate [34].

In conclusion, PCBL without FNAB, under CT fluoroscopy guidance, has an excellent diagnostic accuracy in obtaining a conclusive histologic diagnosis of thoracic lesions and is comparable to FNAB in terms of safety and rates of occurence of complications.

\section{ACKNOWLEDGEMENT}

Declared none.

\section{CONFLICT OF INTEREST}

The authors confirm that this article content has no conflict of interest.

\section{REFERENCES}

[1] Jemal A, Siegel R, Ward E, et al. Cancer statistics, 2009. CA Cancer J Clin 2009; 59: 225-49.

[2] Ferlay J, Autier P, Boniol M, et al. Estimates of the cancer incidence and mortality in Europe in 2006. Ann Oncol 2007; 18: 581-92.

[3] Gupta A, Wallace MJ, Cardella JF, et al. Quality improvement guidelines for percutaneous needle biopsy. J Vasc Interv Radiol 2010; 21: 969-75.

[4] Yamagami T, Iida S, Kato T, et al. Usefulness of new automated cutting needle for tissue-core biopsy of lung nodules under CT fluoroscopic guidance. Chest 2003; 124: 147-54.

[5] Laurent F, Latrabe V, Vergier B, et al. CT-guided transthoracic needle biopsy of pulmonary nodules smaller than $20 \mathrm{~mm}$ : results with an automated 20-gauge coaxial cutting needle. Clin Radiol 2000; 55: 281-7.

[6] Priola AM, Priola SM, Cataldi A, et al. Accuracy of CT-guided transthoracic needle biopsy of lung lesions: factors affecting diagnostic yield. Radiol Med (Torino) 2007; 112: 1142-59.

[7] Moulton JS, Moore PT. Coaxial percutaneous technique with automated biopsy devices: value in improving accuracy and negative predictive value. Radiology 1993; 186: 515-22.

[8] Richardson CM, Pointon KS, Manhire AR, et al. Percutaneous lung biopsies: a survey of UK practice based on 5444 biopsies. Br J Radiol 2002; 75: 731-5.

[9] Manhire A, Carig M, Clelland C, et al. Guidelines for radiologically guided lung biopsy. Thorax 2003; 58: 920-36.

[10] Berquist TH, Bailey PB, Cortese DA, et al. Transthoracic needle biopsy: accuracy and complications in relation to location and type of lesion. Mayo Clin Proc 1980; 55: 475-81.

[11] Stanley JH, Fish GD, Andriole JG, et al. Lung lesions: cytologic diagnosis by fine needle biopsy. Radiology 1987; 162: 389-91.

[12] Lucidarme O, Howarth N, Finet JF, et al. Intrapulmonary lesions: percutaneous automated biopsy with a detachable, 18-gauge, coaxial cutting needle. Radiology 1998; 207: 759-65.

[13] Laurent F, Latrabe V, Vergier B, et al. Percutaneous CT-guided biopsy of the lung: comparison between aspiration and automated cutting needle using a coaxial technique. Cardiovasc Intervent Radiol 2000; 23: 266-72.

[14] Yeow KM, See LC, Lui KW, et al. Risk factors for pneumothorax and bleeding after CT-guided percutaneous coaxial cutting needle biopsy of lung lesions. J Vasc Interv Radiol 2001; 12: 1305-12.

[15] Katada K, Kato R, Anno H, et al. Guidance with real-time CT fluoroscopy: early clinical experience. Radiology 1996; 200: 851-6. 
[16] Hiraki T, Mimura H, Gobara H, et al. Incidence of and risk factors for pneumothorax and chest tube placement after CT fluoroscopyguided percutaneous lung biopsy: retrospective analysis of the procedures conducted over a 9-year period. Am J Roentgenol 2010; 194: 809-14.

[17] Doll R, Hill AB. Smoking and carcinoma of the lung: a preliminary report. Br Med J 1950; 2: 739-48.

[18] Aviram G, Schwartz DS, Meirsdorf S, et al. Transthoracic needle biopsy of lung masses: a survey of techniques. Clin Radiol 2005; 60: 370-4.

[19] Anderson JM, Murchison J, Patel D. CT-guided lung biopsy: factors influencing diagnostic yield and complication rate. Clin Radiol 2003; 58: 791-7.

[20] Geraghty PR, Kee ST, McFarlane G, et al. CT-guided transthoracic needle aspiration biopsy of pulmonary nodules: needle size and pneumothorax rate. Radiology 2003; 229: 475-81.

[21] Gupta S, Krishnamurthy S, Broemeling LD, et al. Small $(</=2-\mathrm{cm})$ subpleural pulmonary lesions: short- versus long- needle-path CTguided biopsy - comparison of diagnostic yields and complications. Radiology 2005; 234: 631-7.

[22] Ohno Y, Hatabu H, Takenaka D, et al. CT-guided transthoracic needle aspiration biopsy of small $(<$ or $=20 \mathrm{~mm})$ solitary pulmonary nodules. AJR Am J Roentgenol 2003; 180: 1665-9.

[23] Tsukada H, Satou T, Iwashima A, et al. Diagnostic accuracy of CT-guided automated needle biopsy of lung nodules. AJR Am J Roentgenol 2000; 175: 239-43.

[24] Wallace MJ, Krishnamurthy S, Broemeling LD, et al. CT-guided percutaneous fine-needle aspiration biopsy of small $(<$ or $=1-\mathrm{cm})$ pulmonary lesions. Radiology 2002; 225: 823-8.

[25] Hiraki T, Mimura H, Gobara $\mathrm{H}$, et al. CT fluoroscopy-guided biopsy of 1,000 pulmonary lesions performed with 20-gauge coaxial cutting needles: diagnostic yield and risk factors for diagnostic failure. Chest 2009; 136: 1612-7.

[26] Poe RH, KallayMC, Wicks CM, et al. Predicting risk of pneumothorax in needle biopsy of the lung. Chest 1984; 85: 232-5.

[27] Saji H, Nakamura H, Tsuchida T, et al. The incidence and the risk of pneumothorax and chest tube placement after percutaneous CTguided lung biopsy: the angle of the needle trajectory is a novel predictor. Chest 2002; 121; 1521-6.

[28] Topal U, Ediz B. Transthoracic needle biopsy: factors effecting risk of pneumothorax. Eur J Radiol 2003; 48: 263-7.

[29] Covey AM, Gandhi R, Brody LA, et al. Factors associated with pneumothorax and pneumothorax requiring treatment after percutaneous lung biopsy in 443 consecutive patients. J Vasc Interv Radiol 2004; 15: 479-83.

[30] Yeow KM, Su IH, Pan KT, et al. Risk factors of pneumothorax and bleeding: multivariate analysis of $660 \mathrm{CT}$-guided coaxial cutting needle lung biopsies. Chest 2004; 126: 748-54.

[31] Yildirim E, Kirbas I, Harman A, et al. CT-guided cutting needle lung biopsy using modified coaxial technique: factors effecting risk of complications. Eur J Radiol 2009; 70: 57-60.

[32] Kazerooni EA, Lim FT, Mikhail A, et al. Risk of pneumothorax in CT-guided transthoracic needle aspiration biopsy of the lung. Radiology 1996; 198: 371-5.

[33] Khan MF, Straub R, Moghaddam SR, et al. Variables affecting the risk of pneumothorax and intrapulmonal hemorrhage in CT-guided transthoracic biopsy. Eur Radiol 2008; 18: 1356-63.

[34] O'Neill AC, McCarthy C, Ridge CA, et al. Rapid needle-out patient-rollover time after percutaneous CT-guided transthoracic biopsy of lung nodules: effect on pneumothorax rate. Radiology 2012; 262: 314-9.

(C) McSweeney et al.; Licensee Bentham Open.

This is an open access article licensed under the terms of the Creative Commons Attribution Non-Commercial License (http://creativecommons.org/licenses/by-nc/3.0/) which permits unrestricted, non-commercial use, distribution and reproduction in any medium, provided the work is properly cited. 